\title{
Influence of the Confinement Factor on the Wavelength-Dependent Output Properties of a Tapered Traveling Wave Semiconductor Amplifier.
}

\author{
A. Gahl and S. Balle
}

\begin{abstract}
We analyze the influence of the confinement factor on the output properties of a tapered traveling wave semiconductor amplifier as a function of the wavelength of the input field and current injected into the amplifier. The interaction of the optical field with the amplifying medium is described by means of a susceptibility function taking into account both the gain and the carrier-induced anti-guiding effect, while the beam propagation method is used to describe the evolution of the field along the amplifier. We find that, for the same input power, devices with lower optical confinement factors allow for the same output optical power with better beam quality and higher saturation power than devices with higher optical confinement factor, in agreement with the available experimental results.
\end{abstract}

High-power semiconductor lasers yielding diffractionlimited beams have attracted a lot of attention because of their promising applications in a wide range of areas like laser printing, free space communications, material processing, optical data storage or pumping of fiber lasers and amplifiers. Since catastrophic optical damage limits the power density of edge-emitting semiconductor lasers to some $10 \mathrm{~mW} / \mu \mathrm{m}$, achieving high output powers $\sim 10 \mathrm{~W}$ requires increasing the width of the active area up to $\sim 100 \mu \mathrm{m}$. However, the output beam of broad-area lasers usually exhibits multiple lateral mode operation, thus degrading the beam quality and leading to filamentation.

Broad-area semiconductor optical amplifiers (SOAs) allow to boost the output power [1], [2] although the lack of lateral structure leads to a degradation of the beam quality in such devices and even to filamentation. SOAs with a tapered gain region have proven very efficient to reach high-output power in a diffraction-limited beam [3], [4], [5] by amplification of the light emitted by a master oscillator, be it integrated or not. Several aspects of these devices have been analyzed both experimentally and theoretically [6], [7], [8], although comparatively little research has been performed on the correlation between the structure of the device and the beam quality [9], [10]. Even lesser attention has been paid to the wavelength dependence of the beam quality in SOAs, although the carrier induced index change through the so-called linewidth enhancement factor - which is highly dependent on both carrier density and wavelength [11], [12] — was pointed out to be as one

This work was supported by the European Union under Project FMRXCT 96-066 and by CICYT, Spain, under Project TIC98-0418.

A. Gahl and S. Balle are with the Dept. de Física Interdisciplinar, Instituto Mediterráneo de Estudios Avanzados, IMEDEA (CSICUIB), E-07071 Palma de Mallorca, Spain. of the main causes of beam degradation [7], [13]. In order to compare the performance of different structures for a fixed amplifier gain, consideration of such a dependence is crucial.

In this paper we numerically analyze by means of a Beam Propagation Method (see e. g. [6], [7], [14]) the influence of the epitaxial structure on the wavelength-dependent beam quality and output power of a tapered traveling-wave SOA. The spectral response of the active medium is included through an explicit form of susceptibility $\chi$ of Quantum Well (QW) media, [15] which allows us to incorporate the carrier dependence of both the gain spectrum and the refractive index change spectrum, thus the carrier and wavelength dependence of the linewidth enhancement factor are directly included in the propagation of the optical field. We compare the output properties of two purely gain-guided SOAs [9], [10] with the same geometrical parameters (see Table I) but the first one having a GRINSCH structure that yields an optical confinement factor $\Gamma=2.4 \%$ (High Optical Confinement, HOC), while the second one has a reduced $\Gamma=1.2 \%$ (Low Optical Confinement, LOC). The active layer of both SOAs consists of a single, $8 \mathrm{~nm}$ thick, $\mathrm{In}_{0.22} \mathrm{Ga}_{0.78} \mathrm{As} \mathrm{QW}$ whose gain peak wavelength is around $1020 \mathrm{~nm}$. In order to simplify the problem, we consider perfect anti-reflection coating on both facets of the SOA, and we also consider that the two SOAs have their active regions at the same temperature, which we suppose to have a uniform distribution in both devices.

Therefore a monochromatic, quasi TE-polarized righttraveling optical field of the form

$$
E=\mathcal{F}(y) \mathcal{E}(x, z) \exp [i(\beta z-\omega t)],
$$

where $\mathcal{F}(y)$ is the transverse-mode profile which has an effective waveguide index $n_{\text {eff }}=c \beta / \omega$, has a longitudinal and lateral profile $\mathcal{E}(x, z)$ given by

$$
\partial_{z} \mathcal{E}=\left(\frac{i}{2 \beta}\left\{\partial_{x}^{2}+\frac{\omega^{2}}{c^{2}}\left[\Delta n^{2}+\Gamma \chi\right]\right\}-\frac{\alpha_{\text {int }}}{2}\right) \mathcal{E} .
$$

In this equation, the field amplitude has been normalized such that $|E|^{2}$ corresponds to photon density, $\alpha_{\text {int }}$ is the internal loss, and $\Gamma$ stands for confinement factor of the optical field to the QW. We have also defined $\Delta n^{2}=n_{\text {pumped }}^{2}-n_{\text {eff }}^{2}$ as the built-in refractive index step of the taper region; henceforth, however, we will for simplicity consider only purely gain-guided tapers, i. e. $\Delta n^{2}=0$. The interaction of the optical field with the carriers injected into the $\mathrm{QW}$ is described by means of the 
electrical susceptibility [15]

$$
\chi=-\chi_{0}\left[2 \ln \left(1-\frac{N / N_{t}}{u+i}\right)-\ln \left(1-\frac{b}{u+i}\right)\right],
$$

where $N_{t}$ is the transparency carrier density, $\chi_{0}$ determines the material gain coefficient $\left(g_{m a t}=\omega \chi_{0} / c n_{e f f}\right)$ and $b$ sets the background index of refraction in the QW layer [15]. In addition, the frequency dependence of the susceptibility is included through

$$
u=\frac{\omega-\omega_{0}}{\gamma}+\sigma\left(\frac{N}{N_{t}}\right)^{1 / 3}
$$

where $\omega_{0}$ is the nominal bandgap frequency, $\gamma$ is the inverse of the polarization dephasing time, and $\sigma\left(N / N_{t}\right)^{1 / 3}$ describes the shrinking of the bandgap as the carrier density is increased [11], [12]. Finally, the boundary conditions for the field are that $\mathcal{E}(x \rightarrow \pm \infty, z) \rightarrow 0$ and $\mathcal{E}(x, z=0)=\mathcal{E}_{i n}(x)$, the input field profile, which we take as a Gaussian of width $W$,

$$
\mathcal{E}_{\text {in }}(x)=\mathcal{E}_{0} \frac{e^{\frac{-x^{2}}{4 W^{2}}}}{(\sqrt{2 \pi} W)^{1 / 2}} .
$$

The steady-state carrier distribution within the QW is in turn given by

$$
0=\frac{\mathcal{I}(x, z)}{e T_{a}}-B N^{2}+D\left[\partial_{x}^{2}+\partial_{z}^{2}\right] N+|\mathcal{E}|^{2} \frac{\omega}{n_{e f f}^{2}} \Im m(\chi)
$$

where $\mathcal{I}(x, z)$ is the distribution of the current per unit area injected into the QW, $e$ is the electron charge in absolute value, $T_{a}$ is the thickness of the QW layer, $B$ is the spontaneous emission coefficient, and $D$ is the ambipolar diffusion coefficient within the QW. The last term in Eq. (4) describes the stimulated emission by interaction of the carriers with the optical field. Finally, the boundary conditions for the carrier density are that

$$
\left[\partial_{z} N\right]_{z=0, L}=0, \quad N(x \rightarrow \pm \infty, z) \rightarrow 0 .
$$

The values of the different parameters in eqs. (1)-(4) are given in Table I. For the current injected into the system, we take it to be uniform in the taper region and zero outside, i. e.,

$$
\mathcal{I}(x, z)=I \frac{\Theta\left(x+x_{t}(z)\right)-\Theta\left(x-x_{t}(z)\right)}{L\left(w_{\text {in }}+w_{\text {out }}\right)},
$$

where $I$ is the total current injected into the system, $L$ is the SOA length, $w_{\text {in }}$ and $w_{\text {out }}$ are the half-widths of the input and output facets of the device, and $x_{t}(z)=$ $w_{\text {in }}+\left(w_{\text {out }}-w_{\text {in }}\right) z / L$ defines the limit of the taper region, whose area is $A=L\left(w_{\text {in }}+w_{\text {out }}\right)$.

In Fig. 1 we show the amplification factor $\left(P_{\text {out }} / P_{\text {in }}\right)$ as a function of wavelength and injection current density $J=I /\left(e A T_{a} B N_{t}^{2}\right)$, for both devices. Amplification factors of the order of $30 \mathrm{~dB}$ can be achieved in the two devices.

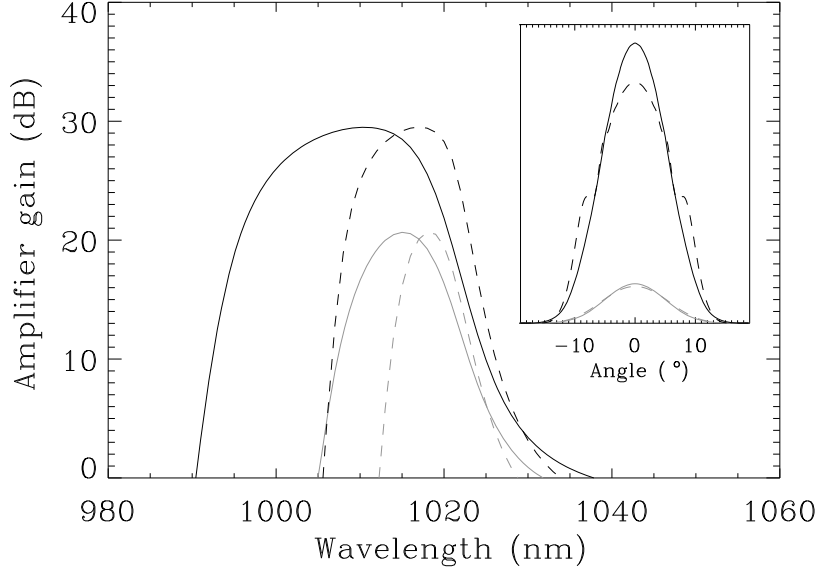

Fig. 1. Wavelength dependence of the amplifier gain of the LOC SOA (solid line) and the HOC SOA (dash line) for a maximum amplifier gain of $29.5 \mathrm{~dB}$ (black) and $20.7 \mathrm{~dB}$ (grey). The input power is $\sim 1 \mathrm{~mW}$. The required current injection levels $J=$ $I /\left(e V_{a} B N_{t}^{2}\right)$ are $J=10.9$ and 4 for the HOC SOA, and $J=36.5$ and 10 for the LOC SOA. The inset shows the far-field profiles of the two devices when operated at the wavelength of maximum amplifier gain in each case.

Obviously, the LOC device requires larger current densities to achieve the same maximum amplification level as the HOC. In addition, the peak amplification factor occurs at a shorter wavelength in the LOC device due to increased band-filling effects associated with the higher current density, which overcome the increased band-gap renormalization in this device, and a larger gain bandwidth is correspondingly achieved. It must be recalled that we have considered that the two devices have the active region at the same temperature; if the two devices were operated at the same substrate temperature, device self-heating would contribute an additional red-shift of the gain-peak wavelength and a material gain reduction in the LOC device that we have not considered. The inset in Fig. 1 shows the far-field profiles of each device when they are operated at the gainpeak. It can be seen that for an amplification factor of $\sim$ $20 \mathrm{~dB}$, both far-field profiles have a clean, almost gaussian shape corresponding to a nearly diffraction-limited output field of $\sim 10^{\circ}$ divergence. However, if we operate the devices at an amplification factor of $\sim 30 \mathrm{~dB}$, the far-field profile of the output from the HOC SOA develops sidelobes that indicate a loss of beam quality, while the LOC device still emits a diffraction limited beam. The reason is that the HOC SOA is already strongly saturated, while the LOC SOA is not yet.

This can be confirmed by doubling the optical input power and evaluating again the amplification factor (see Fig. 2). It can be seen that, when operated at $\sim 30 \mathrm{~dB}$ of peak amplification, the LOC device experiences a reduction of its amplification factor smaller than that of the HOC device, also indicating the higher saturation degree of this device. This reduction is accompanied by an even 


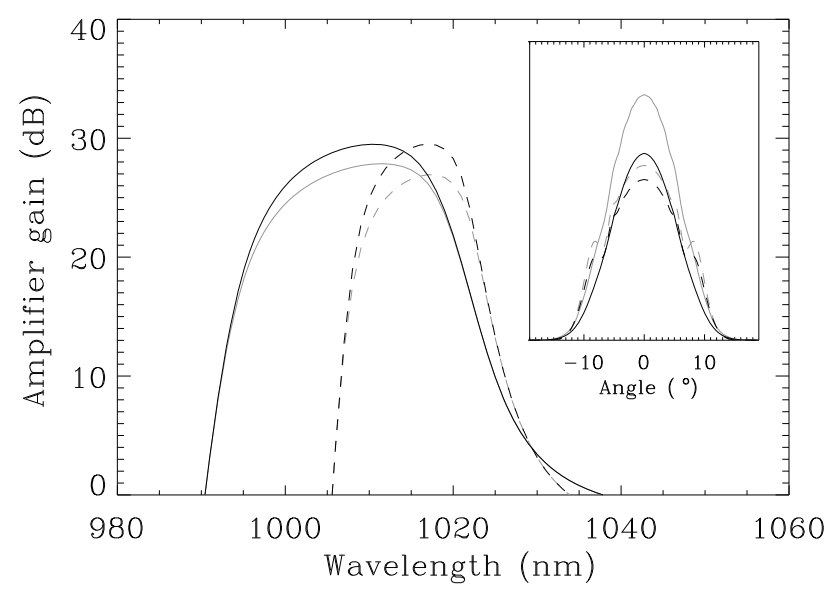

Fig. 2. Wavelength dependence of the amplifier gain of the LOC SOA (solid line) and the HOC SOA (dash line) for different $P_{i n} \sim 1$ $\mathrm{mW}$ (black) and $P_{\text {in }} \sim 2 \mathrm{~mW}$ (grey). Injection currents are $J=36.5$ for the LOC SOA and $J=10.9$ for the HOC SOA The inset shows the far-field profile of the the two devices when operated at the wavelength of maximum amplifier gain in each case.

more noticeable degradation of the far-field profile of the HOC SOA, with enhanced side-lobes as compared to the case where the optical input power was half the actual value (see inset in Fig. 2). These results are in agreement with the results in [9], [10], where it was observed that gain saturation was quite stronger for the HOC SOA than for the LOC SOA.

In conclusion, we have analyzed the influence of the optical confinement factor on the output properties of semiconductor optical amplifiers as a function of wavelength, injection current and input optical power by using the BeamPropagation Method in conjunction with a recently developed approximation for the susceptibility of Quantum-Well media. We have considered two devices which differ only on the degree of confinement of the optical field to the active region, taken to be identical in the two SOAs. The material characteristics are then nominally the same, although the carrier density in both devices is not the same due to the different injection current required to achieve a prescribed amplifier gain. We have found that, although devices with LOC require higher current density to achieve large gain, the beam quality of its output is enhanced as compared to devices with HOC due to a much large saturation power of the former ones, which also renders the beam quality of LOC devices lesser sensitive to the input optical power. Noteworthy, the linewidth enhancement factor is larger in the LOC SOA than in HOC one due to the higher carrier density, but this seems not to determine the degradation of the beam quality. These results are in good qualitative agreement with the experimental results obtained in [9], [10].
Parameter VAlues uSED in the Simulations.

\begin{tabular}{|c|c|c|}
\hline Parameter & Symbol & Value \\
\hline Device length & $\mathrm{L}$ & $2 \mathrm{~mm}$ \\
Input active region half-width & $w_{\text {in }}$ & $5 \mu \mathrm{m}$ \\
Output active region half-width & $w_{\text {out }}$ & $120 \mu \mathrm{m}$ \\
Refractive index step in taper & $\Delta n^{2}$ & 0 \\
Internal loss & $\alpha_{\text {int }}$ & $2 \mathrm{~cm}^{-1}$ \\
Transparency carrier density & $N_{t}$ & $10^{18} \mathrm{~cm}^{-3}$ \\
Susceptibility factor & $\chi_{0}$ & $0.148 \frac{n}{2 \pi}$ \\
Background constant & $b$ & $2000^{-1}$ \\
Bandgap renormalization coefficient & $\sigma$ & 0.2 \\
Polarization dephasing rate & $\gamma$ & $10^{13} \mathrm{~s}^{-1}$ \\
Spontaneous emission coefficient & $B$ & $10^{-27} \mathrm{~cm}^{3} \mathrm{~s}^{-1}$ \\
Ambipolar diffusion coefficient & $D$ & $1 \mathrm{~cm}^{2} \mathrm{~s}^{-1}$ \\
Input field half-width & $W$ & $2 \mu \mathrm{m}$ \\
\hline \multicolumn{2}{|c}{} \\
\hline \multicolumn{2}{|c}{}
\end{tabular}

\section{REFERENCES}

[1] L. Goldberg, D. Mehuys and D. C. Hall, " $3.3 \mathrm{~W}$ cw diffraction limited broad rea semiconductor amplifier", Electron. Lett., vol. 28, pp. 1082-1084, 1992.

[2] L. Goldberg, D. Mehuys, M. R. Surette and D. C. Hall, "Highpower, near-diffraction-limited large-area traveling-wave semiconductor amplifiers", IEEE J. Quantum Electron., vol. 29, pp. 20282043, 1993.

[3] D. Mehuys, L. Goldberg and D. F.Welch, " $5.25 \mathrm{~W}$ cw neardiffraction-limited tapered-stripe semiconductor optical amplifier", IEEE Photon. Technol. Lett., vol. 5, pp. 1179-1182, 1993.

[4] J. P. Donnelly, J. N. Walpole, G. E. Betts, S. H. Grooves, J. D. Woodhouse, F. J. O'Donnell, L. J. Missaggia, R. J. Bailey and A. Napoleone, "High-power $1.3-\mu \mathrm{m}$ InGaAsP/InP amplifiers with tapered gain regions", IEEE Photon. Technol. Lett., vol. 8, pp. 1450-1452, 1996.

[5] R. Parke, D. F. Welch, A. Hardy, R. Lang, D. Mehuys, S. O'Brien, K. Dzurko and D. Scifres, " $2.0 \mathrm{~W}$ CW, diffraction-limited operation of a monolithically integrated master oscillator power amplifier", IEEE Photon. Technol. Lett., vol. 31, pp. 1187-1189, 1995.

[6] R. J. Lang, A. Hardy, R. Parke, D. Mehuys, S. O'Brien, J. Major and D. Welch, "Numerical analysis of flared semiconductor laser amplifiers", IEEE J. Quantum Electron., vol. 29, pp. 2044-2051, 1993.

[7] Z. Dai, R. Michalzik, P. Unger and K. J. Ebeling, "Numerical simulation of broad-area high-power semiconductor laser amplifiers", IEEE J. Quantum Electron., vol. 33, pp. 2240-2254, 1997.

[8] A. Egan, C. Z. Ning, J. V. Moloney, R. A. Indik, M. W. Wright, D. J. Bossert and J. G. McInerney, "Dynamic instabilities in master oscillator power amplifier semiconductor lasers", IEEE J. Quantum Electron., vol. 34, pp. 166-170, 1998.

[9] P. Chazan, S. Morgott, M. Mikulla, R. Kiefer, G. Bihlman, R. Moritz, J. Daleiden, J. Braunstein and G. Weimann, "Influence of the epitaxial layer structure on the beam quality factor of tapered semiconductor amplifiers", LEOS 1997, paper ThP4, pp. 411-412, 1997.

[10] M. Mikulla, P. Chazan, A. Schmitt, S. Morgott, A. Wetzel, M. Walther, R. Kiefer, W. Pletschen, J. Braunstein, and G. Weimann, "High-brightness tapered semiconductor laser oscillators and amplifiers with low-modal gain epilayer-structures", IEEE Photonics Technology Letters, vol. 10, pp. 654-656, 1998.

[11] H. Haug and S. W. Koch, "Quantum theory of the optical and electronic properties of semiconductors", World Scientific (Singapore 1993).

[12] W. W. Chow, S. W. Koch, and M. Sargent III, "Semiconductor Laser Physics" (Springer-Verlag, Berlin, 1994)

[13] J. R. Marciante and G. P. Agrawal, "Nonlinear mechanisms of filamentation in broad area semiconductor lasers", IEEE J. Quantum Electron., vol. 32, pp. 590-596, 1996.

[14] S. Balsamo, F. Sartori, and I. Montrosset, "Dynamic Beam Propagation Method for Flared Semiconductor Power Amplifiers", IEEE J. of Sel. Topics in Quantum Electron., vol. 2, pp. 378-384, 1996

[15] S. Balle, "Simple Analytical Approximations for the Gain and Refractive Index Spectra in Quantum-Well Lasers", Phys. Rev. A 57, pp. 1304-1312 (1998). 